\title{
Miracle cures and embryonic science
}

Anticipation of the therapeutic potential of gene-splicing techniques provided the initial impetus, almost 25 years ago, for the formation of commercial biotechnology. At the time, some may remember, there was a perceptible reluctance to conduct experiments with the new molecular technologies. Although these technologies looked good on paper, the potential risks were sufficient to convince many that genetic engineering should be avoided, even in the laboratory. And all this was voluntary.

Things have certainly changed. In seeking to alleviate diseases that would otherwise be untreatable, biotechnology now takes risks and pushes the limits of what many consider ethical medical research. Although that may be necessary, a result is that experimental therapies frequently are rushed into the clinic, even though the science behind them remains rudimentary. This was true for the first trials of gene therapy in adenosine deaminase-deficient patients. And it was true for septic shock therapies in the early 1990s. Neither met with resounding success.

Just how badly things can go wrong when biotechnology forges ahead regardless of incomplete basic knowledge was demonstrated last month when the results were made public of a trial in which patients with a severe form of Parkinson's disease received human fetal nerve cell transplants (N. Eng. J. Med. 344, 710-719, 2001). The US trial, sponsored by the National Institute for Neurological Disorders and Stroke, lasted for 12 months and followed the progress of 40 patients who had received-through holes drilled in their skulls-dopaminergic brain cells cultured from four human embryos.

Although the study was successful to an extent- the fetal neurons survived in the recipients' brains, they grew out into surrounding brain tissue, and they provided clinical benefit in a small number of younger patients-several patients also paid a tragic price. According to reports in the US and UK media, after a year of doing well, six recipients began to experience "nightmarish" side effects. They started to "writhe, jerk their heads uncontrollably, and throw their arms about involuntarily." One patient was so badly affected he could no longer eat and had to use a feeding tube. Worse still, the side effects were irreversible.

These results are disappointing for the millions of Parkinson's sufferers who looked with promise to fetal cell therapy as a means of ameliorating their condition. The disappointment, however, will be amplified many fold as opponents of human embryo research exploit the disturbing findings to persuade governments to maintain or reintroduce bans on the in vitro manipulation of fetal tissue.

It would be a great pity if this human tragedy were exploited to jeopardize recent legislative progress regarding embryo research. Already, a law passed by the UK government in January allowing the creation of cloned human embryos for the purpose of extending stem cell research is under legal challenge by an antiabortionist group, a situation that is delaying licensing of the technology.

In the United States, President Bush is reappraising the Clinton administration's decision to overturn the ban on fetal tissue research and establish guidelines for the National Institutes of Health to fund related embryonic stem cell research. Predictably, the American Life League's American Bioethics Advisory Commission has cited the "disastrous results" in the Parkinson's study as a major reason for discounting embryonic stem cell research and for derailing the bill S 2015, which is intended to authorize its funding. Christian conservatives are also lobbying loudly for reintroduction of the ban.

As in every situation that involves a tragic occurrence, there is more than sufficient blame to spread around. In this case, the researchers could be accused of proceeding with human studies before even the most rudimentary certainties about Parkinson' disease were known-let alone what the effects of fetal brain implants would be. From our perspective, it is clear that a rigorous evaluation of stem cell biology and its therapeutic potential is needed before human testing of stem cells should continue. In particular, we need to identify the characteristics that define embryonic and adult stem cells and understand the process of differentiation (and dedifferentiation). We need to understand the full potential and limitation of adult cells versus embryonic cells. We need to characterize the chemical and physical signals that dictate how stem cells behave. What's more, we must expand knowledge of anatomical and functional connectivity in the brain and learn how to predict the behavior of stem cells in the brain and the likely success of transplantation strategies.

The lesson applies not only to stem cells, but also to any other experimental treatment under development. On page 289, we report on a US biotechnology company that has not only carried out human experiments in the absence of basic data, but also gone one step farther and completely bypassed the FDA, testing experimental cancer vaccines on subjects in Mexico without regulatory approval. In another example, Italian and US researchers announced in January their intention to clone a human baby from an infertile couple. How exactly they intend to attempt such a feat when our understanding of nuclear transfer is so incomplete, is not clear. The present failure rate of cloning is a staggering $97 \%$. Even when it does work, we don't really understand why or how. One wonders how they would justify the hundreds of wasted eggs, embryos, miscarriages, and abortions, not to mention deformations and stillbirths, expended in the attempt.

There is a saying that one must risk going too far to discover just how far one can go. And biotechnology should continue to strive to develop products rapidly and get them into the clinic. But we must be sure the science is ready. The Parkinson's trial shows the tragic consequences when it isn't. 